\title{
Wall Mechanical Properties and Hemodynamics of Unruptured Intracranial Aneurysms
}

\author{
J.R. Cebral, X. Duan, B.J. Chung, C. Putman, K. Aziz, and A.M. Robertson
}

\begin{abstract}
BACKGROUND AND PURPOSE: Aneurysm progression and rupture is thought to be governed by progressive degradation and weakening of the wall in response to abnormal hemodynamics. Our goal was to investigate the relationship between the intra-aneurysmal hemodynamic conditions and wall mechanical properties in human aneurysms.
\end{abstract}

MATERIALS AND METHODS: A total of 8 unruptured aneurysms were analyzed. Computational fluid dynamics models were constructed from preoperative 3D rotational angiography images. The aneurysms were clipped, and the domes were resected and mechanically tested to failure with a uniaxial testing system under multiphoton microscopy. Linear regression analysis was performed to explore possible correlations between hemodynamic quantities and the failure characteristics and stiffness of the wall.

RESULTS: The ultimate strain was correlated negatively to aneurysm inflow rate $(P=.021)$, mean velocity $(P=.025)$, and mean wall shear stress $(P=.039)$. It was also correlated negatively to inflow concentration, oscillatory shear index, and measures of the complexity and instability of the flow; however, these trends did not reach statistical significance. The wall stiffness at high strains was correlated positively to inflow rate $(P=.014)$, mean velocity $(P=.008)$, inflow concentration $(P=.04)$, flow instability $(P=.006)$, flow complexity ( $P=.019)$, wall shear stress $(P=.002)$, and oscillatory shear index $(P=.004)$.

CONCLUSIONS: In a study of 8 unruptured intracranial aneurysms, ultimate strain was correlated negatively with aneurysm inflow rate, mean velocity, and mean wall shear stress. Wall stiffness was correlated positively with aneurysm inflow rate, mean velocity, wall shear stress, flow complexity and stability, and oscillatory shear index. These trends and the impact of hemodynamics on wall structure and mechanical properties should be investigated further in larger studies.

ABBREVIATIONS: MPM = multiphoton microscope; WSS = wall shear stress

U nderstanding cerebral aneurysm pathogenesis is extremely important for improving aneurysm evaluation and patient management. ${ }^{1-3}$ Numerous previous studies have identified many factors that potentially contribute to the development, enlargement, and rupture of intracranial aneurysms. ${ }^{4}$ However, the links between these factors and the underlying mechanisms responsible for the formation, growth, and stabilization or rupture of cerebral aneurysms is still poorly understood. ${ }^{5,6}$ It is generally

Received October 31, 2014; accepted after revision January 30, 2015.

From the Department of Bioengineering (J.R.C., B.J.C.), Volgenau School of Engineering, George Mason University, Fairfax, Virginia; Department of Mechanical Engineering and Material Science (X.D., A.R.), Swanson School of Engineering, University of Pittsburgh, Pittsburgh, Pennsylvania; Interventional Neuroradiology (C.P.), Inova Fairfax Hospital, Falls Church, Virginia; and Neurosurgery (K.A.), Allegheny General Hospital, Pittsburgh, Pennsylvania.

Please address correspondence to J.R. Cebral, PhD, Bioengineering Department, Volgenau School of Engineering, 4400 University Drive, MSN 2A1, Fairfax, VA 22030; e-mail: jcebral@gmu.edu

- Indicates open access to non-subscribers at www.ajnr.org

http://dx.doi.org/10.3174/ajnr.A4358 accepted that the evolution of cerebral aneurysms is driven by flow-induced progressive degradation of the wall. ${ }^{4,7,8}$ It has been suggested that aberrant aneurysmal flow conditions cause endothelial dysfunction, which induces accumulation of cytotoxic and proinflammatory substances in the wall and thrombus formation that in turn result in the loss of mural cells and wall degeneration. ${ }^{9,10}$ This conjecture is supported by histologic analysis of resected human aneurysm tissue. ${ }^{8}$ Conversely, on the basis of mechanical testing of tissue samples collected after aneurysm clipping, Costalat et $\mathrm{al}^{11}$ conjectured that mechanical stiffness of the aneurysm wall may be associated with rupture. Robertson et $\mathrm{al}^{12}$ recently studied human aneurysm tissues by using a multiphoton microscope (MPM) and found that mechanical properties and collagen architecture can differ, even among unruptured aneurysms. However, the connections between the hemodynamic environment within the aneurysm sac and the wall structure and mechanical behavior have not been studied. 

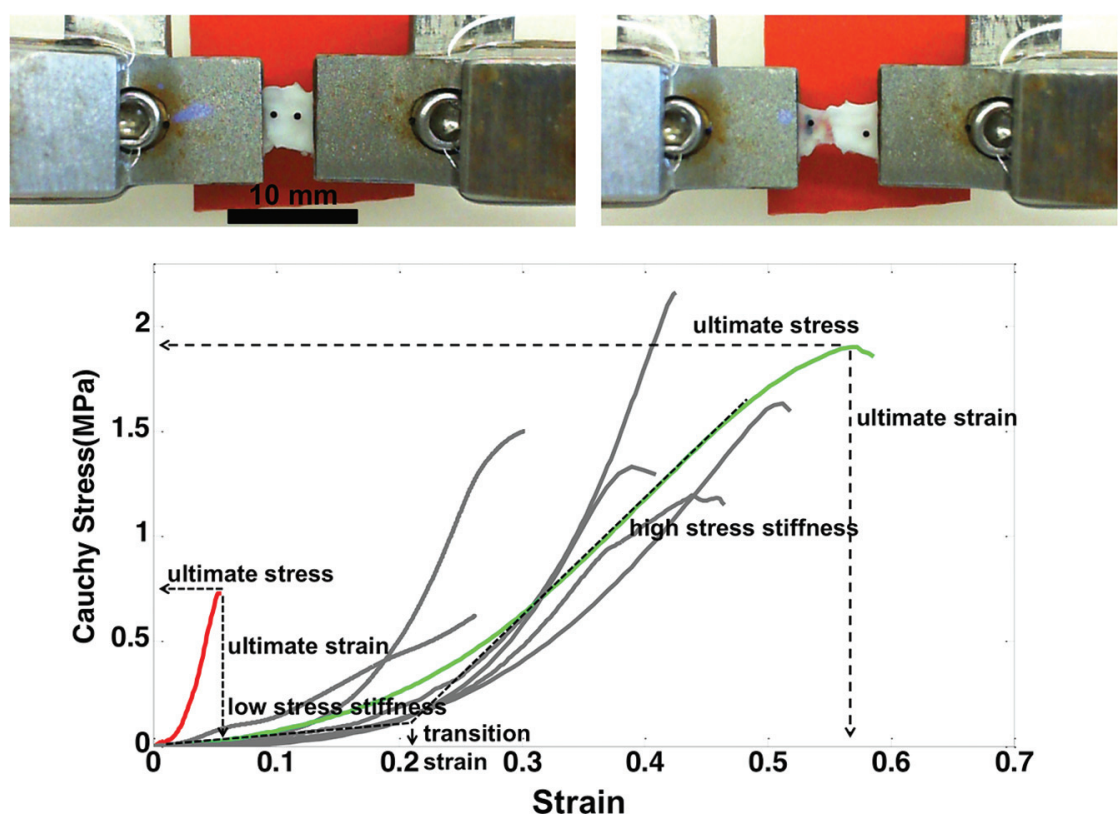

FIG 1. Bottom, Stress-strain relationships. Examples of an aneurysm with stiff walls and low ultimate strain and stress (CA15) and an aneurysm with softer walls and greater ultimate stress and ultimate strain (CA26) are highlighted in red and green, respectively. Top, Picture of a sample with grips before stretching (left) and after it fails (right). Note that the tear occurs near the middle of the sample, not at the grips.

The purpose of this work was to investigate possible associations between aneurysmal hemodynamics and wall mechanical properties in human intracranial aneurysms.

\section{MATERIALS AND METHODS \\ Clinical, Imaging, and Tissue Data}

We analyzed 8 sufficiently large samples (larger than $4 \times 5 \mathrm{~mm}$ ) from a series of 15 harvested unruptured intracranial aneurysm domes in 46 patients who underwent elective surgical clipping and provided consent. Before intervention, each aneurysm was imaged with 3D rotational angiography. During the intervention, after placing the clip, the aneurysm dome was resected. Harvested tissue samples were placed in a prepared vial with $0.9 \%$ (weight/volume) saline solution and transported to the laboratory for analysis within 48 hours. Institutional review board approval was obtained for patient consent, handling of patient data, tissue harvesting, and analysis.

\section{Mechanical Testing of Wall Samples}

The samples were mechanically tested to failure by using a custom-designed uniaxial loading system compatible with a multiphoton microscope that allows simultaneous testing and structural imaging of tissue samples. ${ }^{13}$ In particular, because of their capacity for second harmonic generation, collagen fibers can be imaged without staining and traditional destructive techniques. Collagen fibers in the samples were imaged from the luminal (medial) and abluminal (adventitial) sides by using an FV1000 MPE multiphoton laser scanning microscope (Olympus; Tokyo, Japan) equipped with a DeepSee Mai Tai Ti-Sapphire laser (Spectra-Physics Mountain View, California) with a 1.12 numeric aperture $\times 25$ MPE water-immersion objective at an excitation wavelength of $870 \mathrm{~nm}$. The second harmonic generation signal was collected by using a $400-\mathrm{nm}$ emission filter with a $\pm 50 \mathrm{spec}-$ tral bin. All MPM images shown are in a $500-\times 500-\mu \mathrm{m}$ scale. For mechanical testing, rectangular strips of the aneurysm were cut in the meridional direction (samples from all aneurysms were cut to approximately the same size, $4 \times 7$ $\mathrm{mm}$ ), gripped by mechanical clamps, and placed in a bath of $0.9 \%$ (weight/ volume) saline at room temperature in the uniaxial MPM system. Fine-grade sandpaper was adhered to the grips to avoid the need for adhesives. ${ }^{13}$ Specimens were subjected to uniaxial extension at a speed of $20 \mu \mathrm{m} / \mathrm{s}$, controlled by a linear actuator (ANT-25LA; Aerotech, Pennsylvania), and force was recorded with a 5-lb load cell (MDB-5; Transducer Techniques, Rio Nedo Temecula, California). Force-versus-displacement curves were obtained after 5 cycles of preconditioning to $0.3 \mathrm{~N}$ and used to calculate Cauchy stress as a function of strain. Strain was defined relative to the configuration of the tissue under a load of $0.005 \mathrm{~N}$. Additional details on the tissue-handling protocol, mechanical tests, and MPM imaging were published in a separate article. ${ }^{12}$

\section{Hemodynamics Modeling}

Subject-specific computational fluid dynamics models were constructed from preoperative 3D rotational angiography images. ${ }^{14}$ All aneurysms were located in the anterior circulation. All reconstructions included the internal carotid artery and were extended proximally as much as possible to minimize the effect of inlet boundary conditions on aneurysm hemodynamics. Pulsatile flow conditions were derived from phase-contrast MR measurements in healthy subjects. ${ }^{15}$ Flow waveforms were scaled with the inflow vessel cross-sectional area to achieve a mean wall shear stress (WSS) of $15 \mathrm{dyne} / \mathrm{cm}^{2}$ according to the principle of minimum work (Murray's law). ${ }^{16}$ Vessel walls were assumed rigid, and blood was assumed to be a Newtonian fluid with a density of 1.0 $\mathrm{g} / \mathrm{cm}^{3}$ and viscosity of 0.04 poise. The 3D incompressible NavierStokes equations were numerically solved with finite elements on unstructured grids with a resolution of $0.2 \mathrm{~mm}$ and a time step of 0.01 seconds. $^{14}$

\section{Data Analysis}

A number of variables were computed from the computational fluid dynamics simulations to quantitatively characterize the aneurysm hemodynamic environment, including the aneurysm inflow rate, aneurysm mean velocity, inflow concentration index, POD entropy (a measure of stability of the intra-aneurysmal flow structure), vortex core-line length (a measure of complexity of the intra-aneurysmal flow pattern), shear concentration index (a measure of concentration of the WSS distribution), mean WSS, mean oscillatory shear index, and percent area under low wall shear stress relative to the parent vasculature. All quantities were 
Table 1: Material properties of harvested human aneurysm tissue samples

\begin{tabular}{|c|c|c|c|c|c|c|c|}
\hline Sample No. & $\begin{array}{c}\text { Thickness } \\
\text { (mm) }\end{array}$ & $\begin{array}{l}\text { Transition } \\
\text { Strain }\end{array}$ & $\begin{array}{c}\text { Low-Strain } \\
\text { Stiffness (MPa) }\end{array}$ & $\begin{array}{c}\text { High-Strain } \\
\text { Stiffness (MPa) }\end{array}$ & $\begin{array}{l}\text { Ultimate } \\
\text { Strain }\end{array}$ & $\begin{array}{c}\text { Ultimate } \\
\text { Stress (MPa) }\end{array}$ & $\begin{array}{c}\text { Ultimate } \\
\text { Tension }\left(\mathrm{kg} / \mathrm{s}^{2}\right)\end{array}$ \\
\hline CAl & 0.24 & 0.16 & 0.35 & 12.24 & 0.30 & 1.50 & 285 \\
\hline CA11 & 0.21 & 0.11 & 1.66 & 2.93 & 0.26 & 0.63 & 105 \\
\hline CA12 & 0.20 & 0.28 & 0.58 & 14.09 & 0.42 & 2.16 & 320 \\
\hline CA15 & 0.13 & 0.02 & 2.35 & 24.41 & 0.05 & 0.73 & 90 \\
\hline CA25 & 0.24 & 0.26 & 0.24 & 6.18 & 0.51 & 1.63 & 280 \\
\hline CA26 & 0.45 & 0.22 & 0.90 & 5.43 & 0.57 & 1.90 & 580 \\
\hline CA39 & 0.21 & 0.24 & 0.34 & 6.38 & 0.44 & 1.20 & 183 \\
\hline CA46 & 0.30 & 0.24 & 0.22 & 9.11 & 0.38 & 1.34 & 300 \\
\hline
\end{tabular}

Table 2: Hemodynamic characteristics of cerebral aneurysms

\begin{tabular}{|c|c|c|c|c|c|c|c|c|c|}
\hline Sample No. & $Q(\mathrm{~mL} / \mathrm{s})$ & $\mathrm{ICI}$ & VE $(\mathrm{cm} / \mathrm{s})$ & podent & corelen $(\mathrm{cm})$ & $\mathrm{SCl}$ & WSS (dyne $/ \mathrm{cm}^{2}$ ) & OSI & LSA (\%) \\
\hline CA1 & 1.52 & 2.00 & 9.53 & 0.31 & 2.86 & 4.39 & 19.57 & 0.019 & 49.3 \\
\hline CAll & 0.58 & 0.48 & 6.68 & 0.30 & 1.68 & 11.58 & 11.13 & 0.010 & 79.7 \\
\hline CA12 & 0.71 & 0.65 & 10.18 & 0.62 & 2.84 & 6.00 & 25.30 & 0.025 & 75.5 \\
\hline CA15 & 3.52 & 2.28 & 23.62 & 1.03 & 5.65 & 3.92 & 42.87 & 0.056 & 59.5 \\
\hline CA25 & 0.09 & 0.21 & 0.98 & 0.36 & 0.30 & 21.70 & 1.31 & 0.020 & 97.1 \\
\hline CA26 & 0.61 & 0.70 & 7.24 & 0.29 & 2.80 & 10.22 & 14.17 & 0.016 & 69.1 \\
\hline CA39 & 0.99 & 0.85 & 8.55 & 0.17 & 3.02 & 4.23 & 14.38 & 0.007 & 40.2 \\
\hline CA46 & 2.18 & 1.74 & 14.11 & 0.11 & 1.46 & 2.80 & 23.27 & 0.004 & 62.3 \\
\hline
\end{tabular}

Note:-Corelen indicates vortex core-line length; ICI, inflow concentration index; LSA, area under low wall shear stress relative to the parent vasculature; OSI, mean oscillatory shear index; podent, POD entropy; $Q$, aneurysm inflow rate; $\mathrm{SCl}$, shear concentration index; $\mathrm{VE}$, aneurysm mean velocity; WSS, mean wall shear stress.

calculated as spatial averages over the aneurysm cavity and time averages over the cardiac cycle. For precise mathematical definitions of these variables and a detailed description of the methodology used to compute them, see references 17 and 18 .

The stress-strain curves for the aneurysm wall before failure are largely exponential in nature. ${ }^{12}$ As such, the curves were classified into 1 of 4 regions, listed in order of increasing load (shown in Fig 1): 1) low stress region, in which the wall is highly elastic (toe region), 2) transition region, 3) high-stress region, with a nearly linear response, or 4) subfailure region, marked by a decrease in slope of the stress-strain curve and ending with failure of the tissue. The mechanical response was characterized in terms of the following parameters: 1) low-stress stiffness (slope of a linear fit for the low-stress region), 2) high-stress stiffness (slope of a linear fit for the high-stress region), 3) transition strain (strain at the intersection of the low- and high-stress-stiffness lines), 4) ultimate stress (maximum stress before failure), 5) ultimate strain (the strain corresponding to ultimate stress), and 6) ultimate tension (engineering) (the product of ultimate stress [engineering] and unloaded thickness). The ultimate tension provides a measure of the ability of the wall to withstand loading. In contrast, the ultimate stress and strain are intrinsic material properties that characterize failure behavior. Fiber orientation was quantified in projected stacks of multiphoton images following the method in reference 13 .

Linear regression analysis was performed to explore possible correlations between hemodynamic variables and wall mechanical properties. All correlations were carried out by using the Python package SciPy (http://www.scipy.org/).

\section{RESULTS}

Mechanical behaviors of the aneurysms are listed in Table 1 and Fig 1, and their corresponding flow characteristics are given in Table 2. The correlations for both high-stress stiffness and ultimate strain and hemodynamic variables reached statistical signif-
Table 3: Linear regression of hemodynamic variables against wall ultimate strain and material stiffness at high stress (past the toe region)

\begin{tabular}{llll}
\hline \multicolumn{1}{c}{$\begin{array}{c}\text { Wall Property and } \\
\text { Flow Variable }\end{array}$} & Slope & $\boldsymbol{R}^{\mathbf{2}}$ & $\boldsymbol{P}$ Value \\
\hline Ultimate strain & & & \\
$Q$ & -0.12 & 0.617 & $.021^{\mathrm{a}}$ \\
VE & -0.019 & 0.597 & $.025^{\mathrm{a}}$ \\
ICI & -0.14 & 0.474 & .059 \\
podent & -0.36 & 0.423 & .081 \\
corelen & -0.067 & 0.412 & .086 \\
SCI & 0.011 & 0.186 & .287 \\
WSS & -0.010 & 0.535 & $.039^{\mathrm{a}}$ \\
OSI & -6.44 & 0.414 & .085 \\
LSA & 0.24 & 0.069 & .531 \\
Material stiffness at high stress & & & \\
Q & 5.01 & 0.659 & $.014^{\mathrm{a}}$ \\
VE & 1.20 & 0.717 & $.008^{\mathrm{a}}$ \\
ICI & 6.44 & 0.531 & $.040^{\mathrm{a}}$ \\
podent & 19.9 & 0.741 & $.006^{\mathrm{a}}$ \\
Corelen & 3.48 & 0.629 & $.019^{\mathrm{a}}$ \\
SCI & -0.52 & 0.227 & .232 \\
WSS & 0.51 & 0.813 & $0.002^{\mathrm{a}}$ \\
OSI & 369.3 & 0.772 & $0.004^{\mathrm{a}}$ \\
LSA & -0.101 & 0.065 & 0.53 \\
\hline
\end{tabular}

Note:-Corelen indicates vortex core-line length; $\mathrm{ICI}$, inflow concentration index; LSA, area under low wall shear stress relative to the parent vasculature; OSI, mean oscillatory shear index; podent, $\mathrm{POD}$ entropy; $\mathrm{Q}$, aneurysm inflow rate; $\mathrm{SCl}$, shear concentration index; VE, aneurysm mean velocity; WSS, mean wall shear stress. a Statistically significant.

icance. Table 3 lists the slope of the linear regression, the correlation coefficient $\left(R^{2}\right)$, and the $P$ value of the correlation between each hemodynamic variable and the measured ultimate strain and high-strain stiffness. For a $P$ value of $<.05$, the slope of the corresponding linear regression is significantly different from zero with 95\% confidence.

The relationships between hemodynamic quantities and ultimate strain and high-strain wall stiffness are shown in Figs 2 and 3, respectively. The lines correspond to the straight lines fitted by the linear 

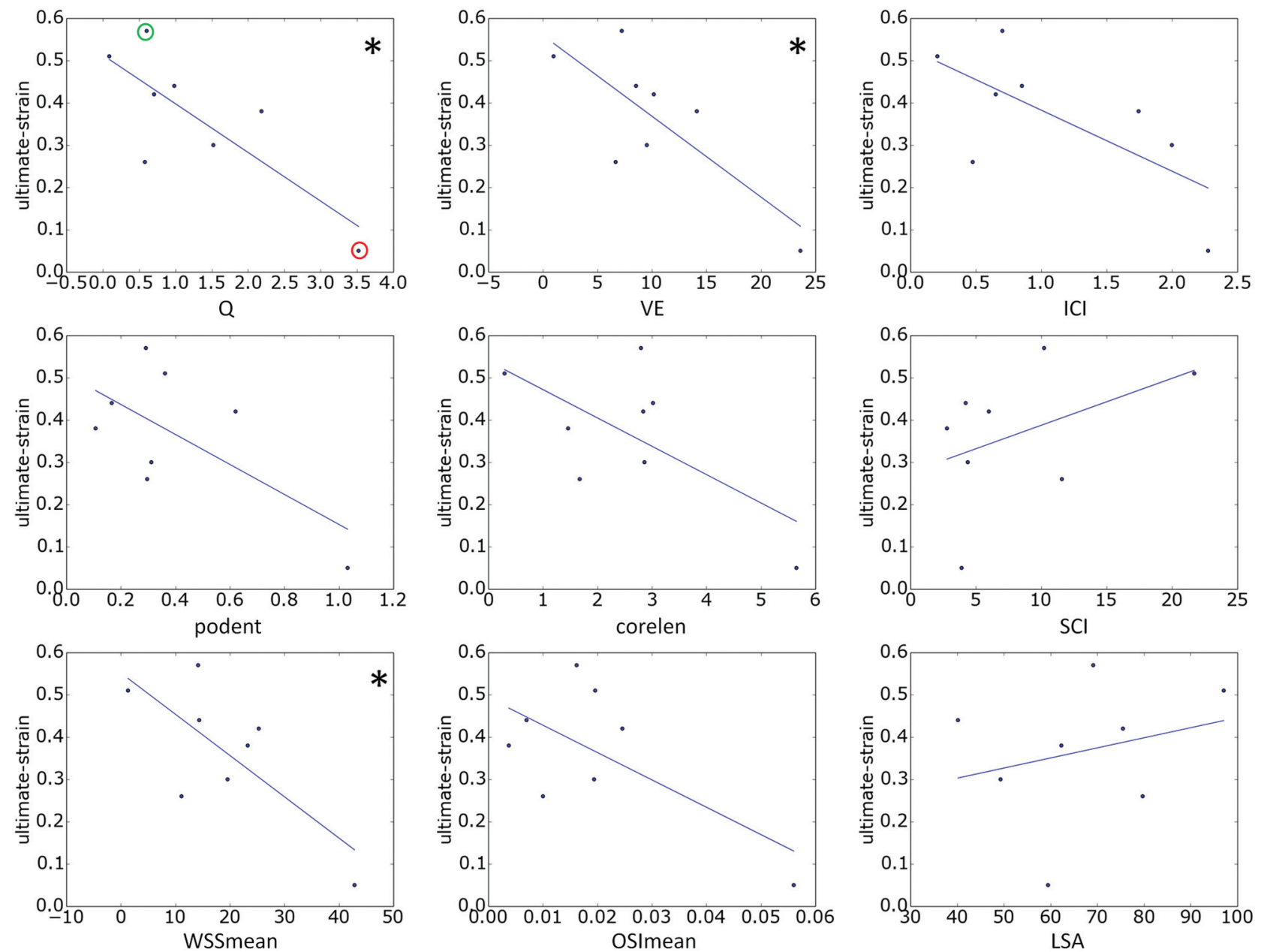

FIG 2. Relationships between hemodynamic variables and ultimate wall strain. Each correlation that reached statistical significance $(95 \%$ confidence) is marked with an asterisk. In the top-left panel, aneurysms CA26 and CA15, exemplified in Figs 4 and 5, are marked with green and red circles, respectively. corelen indicates vortex core-line length; ICI, inflow concentration index; LSA, area under low wall shear stress relative to the parent vasculature; OSI, mean oscillatory shear index; podent, POD entropy; $Q$, aneurysm inflow rate; SCI, shear concentration index (a measure of concentration of the wall shear stress distribution); VE, aneurysm mean velocity; WSS, mean wall shear stress.

regression (slope and intercept provided in Table 3). Each correlation that reached statistical significance is indicated with an asterisk.

The ultimate strain was seen to decrease with increasing aneurysm inflow rate, mean velocity, inflow concentration, flow instability, flow complexity, WSS, and oscillatory shear index; whereas it decreased with increasing concentration of the WSS distribution and area under low WSS. However, only the associations with inflow rate, mean velocity, and mean WSS reached statistical significance. Ultimate stress followed the same trends but did not reach statistical significance (data not shown).

High-stress wall stiffness increased with inflow rate, mean velocity, inflow concentration, flow instability, flow complexity, WSS, and oscillatory shear index, whereas it decreased with the concentration of the WSS distribution and area under low WSS. The relations to velocity, flow instability, WSS, and oscillatory shear index reached statistical significance.

Two aneurysms (CA26 and CA15), representing distinct mechanical behaviors, are considered in greater detail in Fig 4 to illustrate these trends. In particular, with respect to failure properties, the wall of CA26 (Fig 1, green curve, and Figs 2 and 3, green circle) had the largest ultimate strain, largest ultimate tension, and nearly the largest ultimate stress. The walls of CA15 (Fig 1, red curve, and Figs 2 and 3 , red circle) had the lowest ultimate strain, lowest ultimate tension, and second lowest ultimate stress. With regard to the nature of the loading curve, CA26 had nearly the lowest stiffness in the highstress region. In contrast, the walls of CA15 had the lowest transition strain and the largest stiffness in the high-stress region. That is, CA26 was stronger with a softer response at high stress than was CA15.

Flow visualizations of CA26 and CA15 along with MPM images of the collagen fibers of each aneurysm in their unloaded configurations are shown in Fig 4. Isovelocity surfaces (top-left frame of each panel) show a stronger inflow stream and larger intrasaccular velocity in CA15. Flow streamlines (top-center frame of each panel) also show stronger intrasaccular flow penetrating deeper into the cavity of CA15. Vortex core lines (top-right frame of each panel) indicate a more complex and unstable flow pattern within CA15. The WSS distribution (bottom-left frame of each panel) shows higher values and a more irregular WSS distribution in CA15.

The collagen fiber architectures of these 2 aneurysms are qualitatively different. On the luminal (medial) side, CA26 shows a denser distribution resulting in a smoother and more 

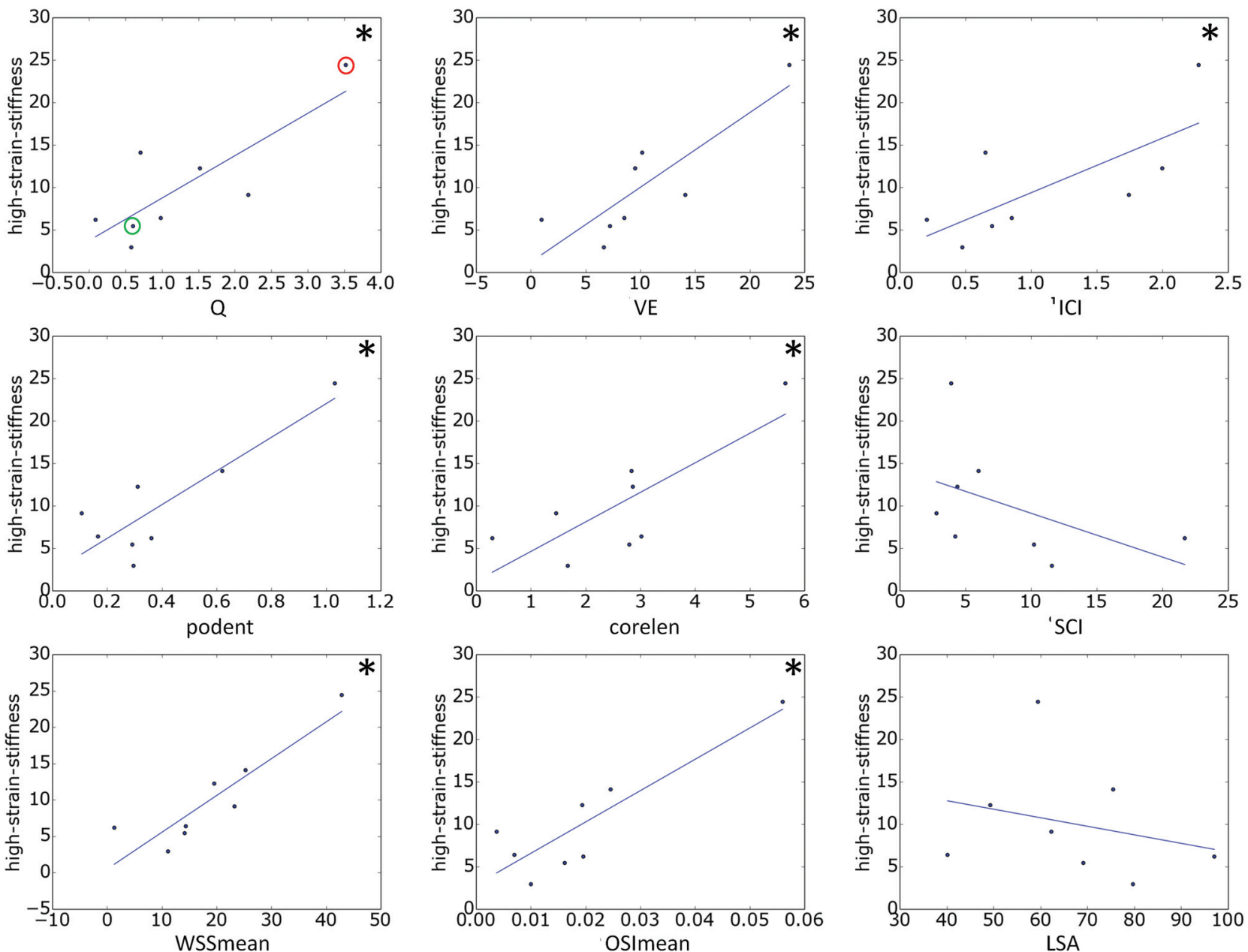

FIG 3. Relationships between hemodynamic variables and wall stiffness at high stress. Each correlation that reached statistical significance $(95 \%$ confidence) is marked with an asterisk. In the top-left panel, aneurysms CA26 and CA15, exemplified in Figs 4 and 5, are marked with green and red circles, respectively. Corelen indicates vortex core-line length; ICl, inflow concentration index; LSA, area under low wall shear stress relative to the parent vasculature; OSImean, mean oscillatory shear index; podent, POD entropy; Q, aneurysm inflow rate; SCl, shear concentration index; VE, aneurysm mean velocity; WSSmean, mean wall shear stress.

homogeneous appearance under the MPM than CA15. On the abluminal (adventitial) side, the fibers of CA26 appear to have more than one preferred orientation direction. The fibers seem to form an interlaced mesh pattern and exhibit a larger degree of waviness. In contrast, the fibers of CA15 close to the abluminal surface appear to follow approximately a single orientation without much interlacing, whereas the fibers deeper in the wall are not unidirectional. Fibers of CA15 exhibit less waviness and, in general, are thicker. The wall thicknesses were 450 $\mu \mathrm{m}$ in CA26 and $130 \mu \mathrm{m}$ in CA15.

\section{DISCUSSION}

Although it is generally accepted that aneurysm evolution is governed by progressive degradation of the wall in response to abnormal hemodynamics, ${ }^{7,8}$ the detailed mechanisms that drive the degeneration and weakening of the wall remain unknown. ${ }^{5}$ Previous studies have identified possible associations between different hemodynamic factors and aneurysmal rupture. ${ }^{4}$ However, it is unclear which parameters are responsible for the mechanisms controlling wall degradation and aneurysm rupture or stabilization. ${ }^{19}$ Determining the influence of intra-aneurysmal hemodynamics on wall structure and strength has the potential to resolve this issue.

A previous study found that rupture site could best be explained in 8 of 9 aneurysms through an association of thinner and stiffer walls with regions of abnormally high WSS. ${ }^{20} \mathrm{Re}-$ sults from our current study seem to support the idea that large flow activity within the aneurysm, as characterized by high velocity and inflow rates; high WSS and oscillatory shear index; and complex unstable flow structures tend to be associated with lower ultimate strains and stiffer walls. Although trends with respect to ultimate stress did not reach statistical significance, they were in agreement with the trends with respect to ultimate strain and warrant further study. In addition, the trends found in this study suggest that increasing the area exposed to low WSS increases the ultimate strain and decreases the high strain stiffness of an aneurysm wall.

In the low-stress region, collagen fibers are found to straighten and reorient as they are recruited to load bearing. ${ }^{12}$ This reorientation can be seen in Fig 5, which displays MPM images of tissue from CA26 under increasing uniaxial loads and corresponding histograms of the distribution of fiber orientations calculated afterward. ${ }^{21}$ In the toe region, fibers straighten as the tissue is loaded. As loading progresses through the transition region, in- 

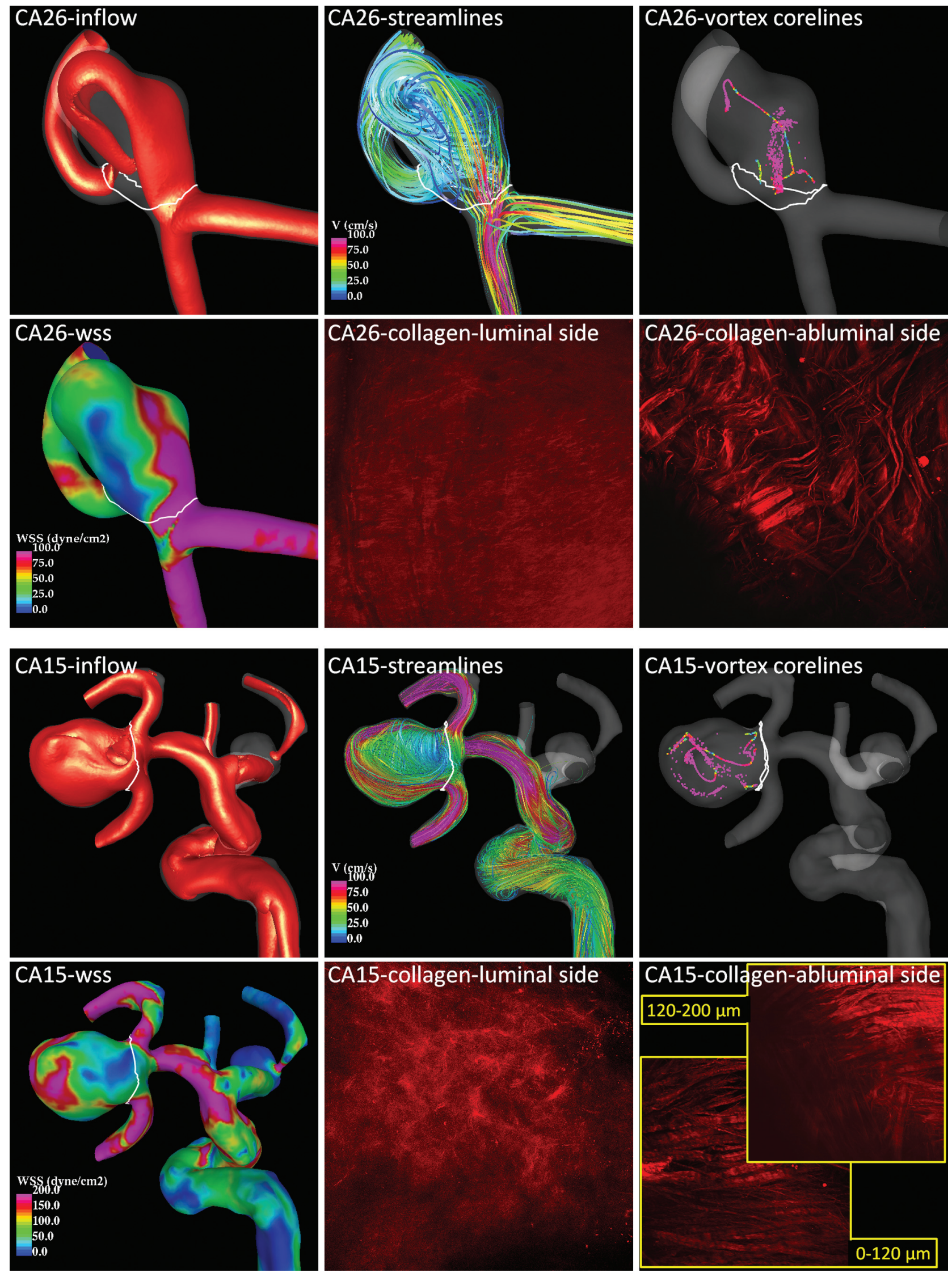

FIG 4. Flow and collagen fibers in 2 example aneurysms. Aneurysm CA26 (top) corresponds to the green circles in Figs 2 and 3 and the green curve in Fig 1, and aneurysm CA15 (bottom) corresponds to the red circles in Figs 2 and 3 and the red curve in Fig 1. 

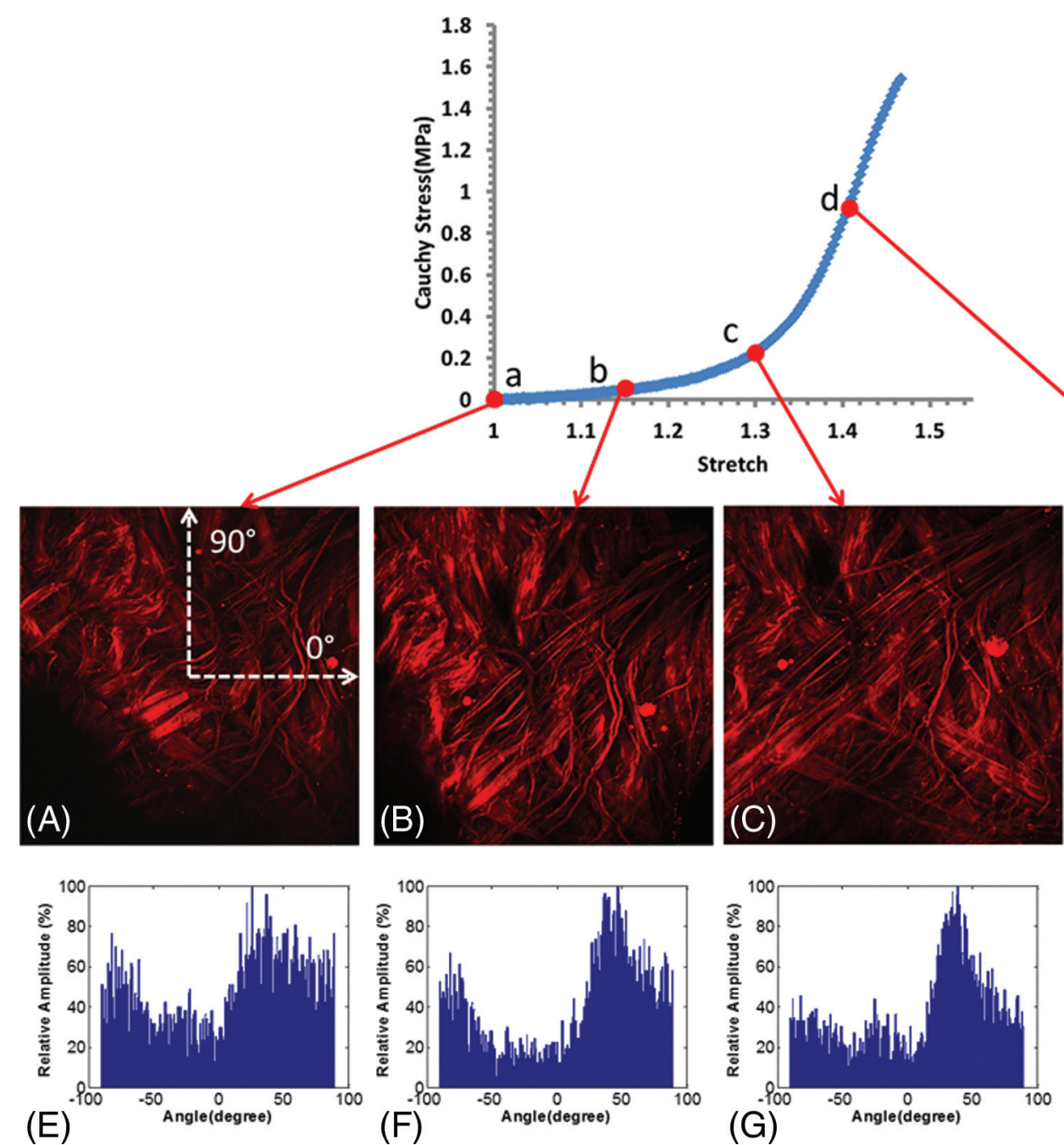

$(\mathrm{G})$
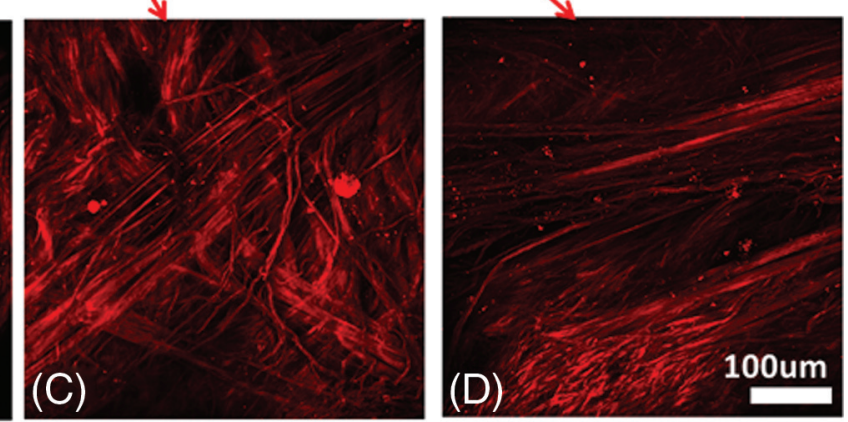

FIG 5. Abluminal view of collagen fiber recruitment during uniaxial loading of aneurysm sample CA26 (green in Figs 1-3) obtained by using the uniaxial MPM system. The images were obtained at stretches of $1.0(A), 1.15(B), 1.3(C)$, and $1.4(D)$ and were formed from a projection of stacks over an approximately $95-\mu \mathrm{m}$ depth of tissue. $E-H$, Histograms of fiber-orientation distribution of the MPM images at stretches of 1.0, 1.15, 1.3, and 1.4, respectively. The horizontal direction on the image is $0^{\circ}$, and the vertical direction is $90^{\circ}$ (as shown in $A$ ).

creasing numbers of fibers are straightened and recruited to load bearing. Finally, in the high-stress region, small changes in stretch require large increases in load, consistent with the extensive state of recruitment of the collagen fibers. Hence, the high-strain stiffness of the tissue reflects the properties of recruited fibers. Stiffer behavior in the aneurysm wall would suggest a higher density of collagen fibers or a stiffer collagen fabric, perhaps caused by increased cross-linking. The larger waviness of collagen fibers in CA26 may explain its increased toe region relative to CA15, which showed little waviness. The magnitude of ultimate strain is influenced by a number of factors, including the size of the toe region, high-stress stiffness, and ultimate stress. CA15 failed at a lower strain relative to that of other samples, consistent with the combined effects of a smaller toe region, greater high-strain stiffness, and lower ultimate stress. The increased stiffness at high stress in this sample is consistent with a higher density of abluminal collagen fibers. In contrast, CA26 failed at a higher strain, which is consistent with its longer toe region, lower high-strain stiffness, and greater ultimate stress.

Our results suggest that the hemodynamic environment within the aneurysm sac influences the mechanical behavior of the aneurysm wall, which in turn strongly depends on the architecture and quality of collagen fibers. The direct connection between hemodynamic conditions and collagen architecture has not yet been explored and is the focus of our ongoing research.

We assessed the connection between global hemodynamic variables and wall mechanical and failure properties. Such global hemodynamic characteristics are appealing, because they are less sensitive to local geometric features. Although this is a sound approach, local flow properties may cause focal changes to the wall in some aneurysms, which in principle can be the cause of failure. Although some of the hemodynamic parameters considered here, such as shear concentration index, reflect local features, the analysis was not performed locally. In the future, it would be valuable to find ways to directly map local mechanical properties to local hemodynamics to extend the current study.

In this study we considered unruptured aneurysms. Although in this study we focused on unruptured aneurysms, the unruptured population seems to harbor a subpopulation of aneurysms vulnerable to rupture. ${ }^{12}$ Thus, studies of already ruptured aneurysms would be valuable to determine if the vulnerable subpopulation and ruptured aneurysms share the same trends identified in this study. 
Among ruptured aneurysms, some are known to include a subpopulation with thin hypocellular walls devoid of endothelial cells. ${ }^{22}$ Hence, this subpopulation of aneurysms may represent a later stage of aneurysm progression in which the role of hemodynamics is fundamentally different from that for the unruptured aneurysm population.

The current study suffers from some limitations. The trends reported here need to be confirmed with larger datasets. A number of idealizations, such as modeling the aneurysm wall as rigid and blood as a single-phase fluid with constant viscosity, were imposed in the hemodynamics studies. Our flow conditions were derived from healthy subjects and scaled with patient-specific inflow vessel sizes, but the effects of comorbidities such as hypertension were not considered. Although our assumptions may have been reasonable, ${ }^{23}$ the effects of other comorbidities should be investigated in future studies. In addition, we considered uniaxial loading, recognizing that in vivo loading is closer to equibiaxial. This choice was motivated largely by the desire to test the tissues to failure coupled with the small size of the aneurysm samples. Nevertheless, to our knowledge, our preliminary results are the first to show the link between hemodynamics and aneurysm wall properties and suggest several provocative hypotheses about the relationship between in vivo hemodynamics, wall structure, and wall mechanical properties that need to be tested with larger series of human aneurysm tissue samples.

\section{CONCLUSIONS}

In unruptured intracranial aneurysms, wall ultimate strain was correlated negatively with aneurysm inflow rate, mean velocity, and mean wall shear stress. Wall stiffness in the high-stress region was positively correlated with aneurysm inflow rate, mean velocity, wall shear stress, flow complexity, and in stability.

\section{ACKNOWLEDGMENTS}

This work was supported by National Institutes of Health grant R21 NS080031.

Disclosures: Juan Cebral—RELATED: Grant: National Institutes of Health (NIH); Support for Travel to Meetings for the Study or Other Purposes: University of Pittsburgh, Comments: Paid for hotel for research visits; UNRELATED: Grants/Grants Pending: $\mathrm{NIH}^{\star}$ and Philips, ${ }^{*}$ Comments: Research grants. Xinjie Duan-RELATED: Grant: NIH grant R21 NS080031.* Christopher Putnam-UNRELATED: Consultancy: Codman Neurovascular; Payment for Lectures (including service on speakers bureaus): Codman Neurovascular, Comments: Annual fellow training program. Anne Robertson—RELATED: Grant: NIH grant 1R21 NS080031-01, * Comments: The submitted paper is based on work performed as part of this NIH R21 grant. Support for Travel to Meetings for the Study or Other Purposes: NIH grant IR21 NS080031-01, travel to the Intracranial Stent Meeting, and to the World Congress of Biomechanics. UNRELATED: Consultancy: NIH, Comments: served on a study section for the $\mathrm{NIH}$; Grants/Grants Pending: NIH, Travel/Accommodations/Meeting Expenses Unrelated to Activities Listed: travel support for research visit to Oxford University and the University of Sheffield. Travel support for a presentation at Cerebrovascular Rounds, University of California at San Francisco Medical Center. Travel support to the 12th Congress of the World Federation of Interventional and Therapeutic Neuroradiology and the International Intracranial Stent Meeting. Travel support for the 51st Annual Meeting of the American Society of Neuroradiology; Other: Fees for serving on a review panel for $\mathrm{NIH}$. *Money paid to the institution.

\section{REFERENCES}

1. Francis SE, Tu J, Qian Y, et al. A combination of genetic, molecular and haemodynamic risk factors contributes to the formation, en- largement and rupture of brain aneurysms. J Clin Neurosci 2013;20: 912-18 CrossRef Medline

2. Lall RR, Eddleman CS, Bendok BR, et al. Unruptured intracranial aneurysms and the assessment of rupture risk based on anatomical and morphological factors: sifting through the sands of data. $\mathrm{Neu}$ rosurg Focus 2009;26:E2 CrossRef Medline

3. Weir B, Macdonald RL. Intracranial aneurysms and hemorrhage: an overview. In Neurosurgery, Wilkins RH, Rengachary SS, eds. New York: McGraw-Hill; 1996:2191-213

4. Cebral JR, Raschi M. Suggested connections between risk factors of intracranial aneurysms: a review. Ann Biomed Eng 2013;41:1366-83 CrossRef Medline

5. Sforza DM, Putman CM, Cebral JR. Hemodynamics of cerebral aneurysms. Annu Rev Fluid Mech 2009;41:91-107 CrossRef Medline

6. Meng H, Tutino VM, Xiang J, et al. High WSS or low WSS? Complex interactions of hemodynamics with intracranial aneurysm initiation, growth, and rupture: toward a unifying hypothesis. AJNR Am J Neuroradiol 2014;35:1254-62 CrossRef Medline

7. Humphrey JD, Canham PB. Structure, mechanical properties, and mechanics of intracranial saccular aneurysms. J Elasticity 2000;61: 49-81 CrossRef

8. Frösen J, Tulamo R, Paetau A, et al. Saccular intracranial aneurysm: pathology and mechanisms. Acta Neuropathol 2012;123:773-86 CrossRef Medline

9. Ujiie $H$, Tachibana $H$, Hiramatsu $O$, et al. Effects of size and shape (aspect ratio) on the hemodynamics of saccular aneurysms: a possible index for surgical treatment of intracranial aneurysms. Neurosurgery 1999;45:119-29; discussion 129-30 CrossRef Medline

10. Frsen J. Smooth muscle cells and the formation, degeneration, and rupture of saccular intracranial aneurysm wall-a review of current pathophysiological knowledge. Transl Stroke Res 2014;5:347-56 CrossRef Medline

11. Costalat V, Sanchez M, Ambard D, et al. Biomechanical wall properties of human intracranial aneurysms resected following surgical clipping (IRRAs Project). J Biomech 2011;44:2685-91 CrossRef Medline

12. Robertson AM, Duan X, Aziz KM, et al. Diversity in the strength and structure of unruptured cerebral aneurysms. Ann Biomed Eng 2015; 43:1502-15 CrossRef Medline

13. Hill MR, Duan X, Gibson GA, et al. A theoretical and non-destructive experimental approach for direct inclusion of measured collagen orientation and recruitment into mechanical models of the artery wall. J Biomech 2012;45:762-71 CrossRef Medline

14. Cebral JR, Castro MA, Appanaboyina S, et al. Efficient pipeline for image-based patient-specific analysis of cerebral aneurysm hemodynamics: technique and sensitivity. IEEE Trans Med Imaging 2005;24:457-67 CrossRef Medline

15. Ford MD, Alperin N, Lee SH, et al. Characterization of volumetric flow rate waveforms in the normal internal carotid and vertebral arteries. Physiol Meas 2005;26:477-88 CrossRef Medline

16. Cebral JR, Castro MA, Putman CM, et al. Flow-area relationship in internal carotid and vertebral arteries. Physiol Meas 2008;29:585-94 CrossRef Medline

17. Mut F, Löhner R, Chien A, et al. Computational hemodynamics framework for the analysis of cerebral aneurysms. Int J Numer Method Biomed Eng 2011;27:822-39 CrossRef Medline

18. Byrne G, Mut F, Cebral J. Quantifying the large-scale hemodynamics of intracranial aneurysms. AJNR Am J Neuroradiol 2014;35: 333-38 CrossRef Medline

19. Kallmes DF. Point: CFD-computational fluid dynamics or confounding factor dissemination. AJNR Am J Neuroradiol 2012;33: 395-96 CrossRef Medline

20. Cebral JR, Vazquez M, Sforza DM, et al. Analysis of hemodynamics and wall mechanics at sites of cerebral aneurysm rupture. J Neurointerv Surg 2015;7:530-06 CrossRef Medline 
21. Schriefl AJ, Reinisch AJ, Sankaran S, et al. Quantitative assessment of collagen fibre orientations from two-dimensional images of soft biological tissues. $J$ R Soc Interface 2012;9:3081-93 CrossRef Medline

22. Frösen J, Piippo A, Paetau A, et al. Remodeling of saccular cerebral artery aneurysm wall is associated with rupture: histological anal- ysis of 24 unruptured and 42 ruptured cases. Stroke 2004:35;2287-93 CrossRef Medline

23. Sarrami-Foroushani A, Villa-Uriol MC, Nasr Esfahany M, et al. Modeling of the acute effects of primary hypertension and hypotension on the hemodynamics of intracranial aneurysms. Ann Biomed Eng 2015;43:207-21 CrossRef Medline 